\title{
Kernos
}

Revue internationale et pluridisciplinaire de religion grecque antique

20 | 2007

Varia

\section{GIANNISI Phoebé, Récits des voies. Chant et cheminement en Grèce archaïque}

\section{André Motte}

URL : https://journals.openedition.org/kernos/334

DOI : $10.4000 /$ kernos.334

ISSN : 2034-7871

\section{Éditeur}

Centre international d'étude de la religion grecque antique

\section{Édition imprimée}

Date de publication : 1 janvier 2007

Pagination : 406-407

ISSN : 0776-3824

Référence électronique

André Motte, « GiAnnisI Phoebé, Récits des voies. Chant et cheminement en Grèce archaïque », Kernos [En ligne], 20 | 2007, mis en ligne le 17 mars 2011, consulté le 08 septembre 2022. URL : http:// journals.openedition.org/kernos/334; DOI : https://doi.org/10.4000/kernos.334 
représentations de figurines sur trépied qu'il avait d'abord considérées comme des images de guerrier ou de kouros (p. 72 et 191; p. 83-84 et 202).

L'ouvrage n'est donc pas exempt de faiblesses et de défauts. Mais il faut lui reconnaittre le mérite de mettre en lumière, autour d'un corpus de bronzes que personne n'avait jusqu'ici identifié comme un ensemble cohérent, un très riche ensemble d'aperçus et de questions touchant aux pratiques sociales et rituelles, à la relation entre productions artistiques et valeurs sociales, à l'articulation entre objets, chant et écriture, qui ouvrent beaucoup de perspectives nouvelles et de réflexions extrêmement stimulantes pour la compréhension de la société grecque de la fin de l'époque géométrique et du haut archaïsme. C'est cette impression de stimulation et d'enrichissement qui domine largement à la fin de la lecture.

François de Polignac

(Centre Louis Gernet [EHESS/CNRS], Paris)

GIANNISI Phoebé, Récits des voies. Chant et cheminement en Grèce archä̈que, Grenoble, Jérôme Millon, 2006. 1 vol. $13,5 \times 21 \mathrm{~cm}, 190$ p. (coll. Horos). ISBN : 2-84137-202-2.

Si vous aimez sortir des sentiers battus ou, plus exactement, revisiter avec un regard neuf des chemins bien connus comme le sont certaines voies sacrées menant à d'illustres sanctuaires, mais aussi les chemins métaphoriques en lesquels se transforment parfois les chants de certains poètes, ne manquez pas de lire cet ouvrage. Il s'agit de la version remaniée d'une thèse de doctorat soutenue, en 1994, à Lyon II (Langues, Histoire et Civilisations des Mondes Anciens) par une diplômée en architecture qui enseigne aujourd'hui à l'École d'Architecture de l'Université de Thessalie (Volos). Le livre devait être préfacé par P. Vidal-Naquet, décédé peu avant la publication; il l'est par J. Svenbro qui fut, lui aussi, membre du jury.

L'idée originale de l'ouvrage, on l'aura deviné, est d'explorer les riches analogies qu'a tissées la Grèce archaïque entre le chemin et le chant, entre la voie et la voix, si l'on préfère. Qu'a-t-on bien pu pressentir de commun à l'époque entre les voies construites et les poèmes ? Pour répondre à la question, l'A. examine tout d'abord, dans une première partie, les voies d'accès menant au temple de quelques grands sanctuaires extra-urbains, celui d'Héra à Samos et ceux d'Apollon à Didymes, à Délos et à Ptôon en Béotie. En ces lieux et en d'autres, les fouilles ont manifesté la présence de nombreuses constructions et sculptures érigées en offrandes, des kouroi notamment; à la différence de celles que l'on trouve aux abords des temples égyptiens, ces offrandes étaient souvent singularisées et propres dès lors à évoquer des souvenirs particuliers et à déclencher des récits dans l'esprit des passants. L'étude des termes utilisés pour désigner ces offrandes (anathèmata, agalmata, sèmata) ainsi que les inscriptions qui y sont gravées témoignent pareillement du but narratif qu'implique leur dépôt en ces endroits. La seconde partie s'attache à montrer les fils qui relient chants et chemins dans la littérature grecque des premiers siècles, à commencer par les emplois des mots oimos et oimè qui véhiculent cette double signification. L'A. consacre aussi un intéressant chapitre à la mnémotechnique antique qui fait du parcours l'essence de la remémoration et l'élément principal des procédés auxquels elle fait appel (cf. les "voies de la mémoire », nous dirions aussi aujourd'hui « les territoires »), elle explore les notions de trace, de pas, de mètre et de rythme, évoque les procédés de la généalogie et du catalogue (sont ici rencontrées des questions qu'abordent aussi les Actes du dernier congrès du CIERGA publiés dans Kernos 19), fait une analyse de deux itinéraires tracés dans l'Odyssée, des divers cheminements que dessine l'Hymne homérique à Apollon et de riches métaphores du chant et du chemin que déploie Pindare. La conclusion élargit plus encore l'éventail en évoquant notamment la thèse anthropologique de la marche humaine considérée comme la source de la production technique, de la langue, de la voix et de la formation d'une mémoire. 
Le dernier mot est pour affirmer modestement que ce livre n'est qu'« un prologue, l'ouverture d'un chemin à suivre, mais déjà tracé depuis des siècles ». On ne pouvait mieux dire. Si beaucoup de perspectives sont ouvertes ici, il arrive de fait qu'elles soient parfois rapidement refermées. La matière est d'ailleurs immense, comme l'A. l'a bien pressenti. Au passage, elle évoque, par exemple, le poète-philosophe Parménide qui est aussi de cette époque et l'usage symbolique qu'il fait de l'image du chemin; il eût fallu à tout le moins citer l'ouvrage de L. Couloubaritsis (Mythe et philosophie chez. Parménide, Bruxelles, 1990²) dont l'interprétation fait une place décisive à ce symbole. Les éléments de réflexion que Ph.G. propose sont de nature à enrichir grandement le traitement du thème pèlerin en Grèce. À l'inverse d'ailleurs, elle aurait pu tirer elle-même profit d'études déjà parues sur le sujet; on pense notamment aux théories que les Athéniens envoyaient à Délos et à Delphes, sur les traces respectivement d'un héros ou d'un dieu ( $f$., par ex., G. Siebert, dans Les Pèlerinages dans l'Antiquité classique et biblique, Paris, Geuthner, 1973, p. 33-53, et ma contribution dans Histoire des pèlerinages non chrétiens, Paris, Hachette, 1987, p. 94-135). Pour d'autres matières, la bibliographie est en revanche étoffée (environ 230 titres au total). Un index eût été bien utile. Mais saluons avant tout l'originalité de l'entreprise et son audace pluridisciplinaire.

André Motte

(Université de Liège)

CALAME Claude, Pratiques poétiques de la mémoire. Représentations de l'espace-temps en Grèce ancienne, Paris, La Découverte, 2006. 1 vol. 13,5 × $22 \mathrm{~cm}, 322$ p. (Textes à l'appui. Série Histoire classique). ISBN : 2-7071-4798-1.

Comme l'indique le sous-titre, cet ouvrage est une réflexion sur les représentations grecques de l'espace et du temps. Toutefois, contrairement aux perspectives philosophiques qui ont souvent marqué de telles investigations, c'est une analyse des pratiques discursives de différents matériaux poétiques qui est ici privilégiée. Il s'agit, par l'analyse de quatre " configurations textuelles » particulières, de comprendre "la représentation discursive du temps dans sa logique propre et en relation avec l'espace de son développement» (p. 83). L'approche méthodologique se déploie dans un long chapitre introductif qui pose également le cadre des manières contemporaines d'aborder les concepts d'espace et de temps. C.C. offre tout d'abord une critique, brève mais incisive, de l'approche heideggerienne du temps. Ensuite, dans le cadre de l'analyse, exigeante, de son appropriation et de son dépassement par P. Ricœur, c'est la question de l'histoire qui est posée. L'histoire est à la fois savoir partagé sur le passé, pratique discursive, et perception et représentation du passé, ces trois modalités de l'histoire se trouvant en perpétuelle interaction. En effet, l'histoire comme savoir se nourrit de la représentation du passé et le savoir partagé ne reste pas imperméable au remodelage discursif de l'histoire comme pratique. À cet égard, le domaine grec est tout particulièrement intéressant puisque nous situons là les premiers moments d'une histoirepratique discursive dont l'A. souligne l'intimité avec la conception classique de la fabrique poétique. Cette intimité n'est pas contredite, comme on l'affirme souvent, par le célèbre passage d'Aristote comparant poésie et histoire (Poétique, 1451b 27-32, commenté p. 62-63). Dès lors, l'historiographie devient une « historio-poiésis » et l'espace est aussi important que le temps dans l'activité « configurante» des premiers logographes. Mais la dimension énonciative de la mise en discours historiographique n'est pas déterminante seulement pour appréhender le travail de «l'historiopoiète » grec : nos pratiques contemporaines n'y échappent pas et la conscience de cette complexité permet de contextualiser - et de relativiser aussi nos productions d'aujourd'hui en évitant de les figer en savoirs immuables... C'est donc la construction symbolique et discursive du temps et de l'espace qui est étudiée dans 